\title{
Determinação da curva de intensidade-duração-frequência do município de Cruzeiro do Sul - Acre
}

\author{
Determination of the intensity-duration-frequency curve of the \\ municipality of Cruzeiro do Sul - Acre
}

\author{
Nayara dos Santos Albrigo ${ }^{(\mathbb{D})}$, Maylla Tawanda dos Santos Pereira' ${ }^{1}$, Nelma

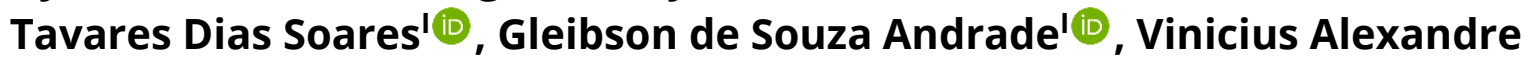 \\ Sikora de Souza"(i), Ana Lúcia Denardin da Rosa"(i) \\ 'Fundação Universidade Federal de Rondônia, Porto Velho, RO, Brasil \\ "Universidade Federal do Rio de Janeiro, Rio de Janeiro, RJ, Brasil
}

\section{RESUMO}

Informações de eventos pluviométricos extremos associados com previsibilidades e probabilidades, principalmente em curvas de intensidade-duração-frequência (IDF), são essenciais para o desenvolvimento de projetos de engenharia voltados ao saneamento, à drenagem e impermeabilização de superfícies, que permitem oferecer condições mais adequadas para dimensionamento de obras e serviços da natureza hidráulico-hidrológica. Contudo, grande parte da Região Norte do país não possui essa informação disponível ou atualizada. Assim, o objetivo desse estudo foi desenvolver a equação IDF do município de Cruzeiro do Sul - AC. Utilizou-se série histórica de 14 anos distribuídos entre 1993 e 2011, tais dados foram analisados pela distribuição de Gumbel, sendo os mesmos relacionados, por meio da desagregação de chuva diária, para períodos de retorno compreendendo 2 a 100 anos e durações pluviométricas de 5 minutos a 24 horas. Nas análises para a construção da curva, observou-se que os anos 1995 e 2002 corresponderam aos anos com maiores índices de altura precipitada, sendo de $111 \mathrm{~mm}$ e $103 \mathrm{~mm}$, respectivamente, contudo esses eventos apresentaram tempo de retorno estimado entre 3 e 8 anos, o que não denota eventos anômalos. A curva IDF construída no estudo apresentou boa aderência aos dados observados, o que comprova o uso da mesma na região.

Palavras-chave: Equação IDF; Precipitação máxima; Probabilidade de Gumbel

\section{ABSTRACT}

Information on extreme rainfall events associated with predictability and probabilities, especially in intensityduration-frequency (IDF) curves, are essential for the development of engineering projects aimed at sanitation, drainage and waterproofing of surfaces, which allow to offer more suitable conditions for dimensioning hydraulic and hydrological works and services. However, much of the North Region of the country does not have this information available or updated. Thus, the objective of this study was to develop 
the IDF equation for the municipality of Cruzeiro do Sul - AC. A 14-year historical series was used, distributed between 1993 and 2011, such data were analyzed by the Gumbel distribution, the same being related, by means of the daily rain breakdown, for return periods comprising 2 to 100 years and rainfall durations of 5 minutes to 24 hours. In the analysis for the construction of the curve, it was observed that the years 1995 and 2002 corresponded to the years with the highest precipitated height indexes, being $111 \mathrm{~mm}$ and 103 $\mathrm{mm}$, respectively, however these events had an estimated return time between 3 and 8 years, which does not denote anomalous events. The IDF curve constructed in the study showed good adherence to the observed data, which proves its use in the region.

Keywords: IDF equation; Maximum rainfall; Gumbel probability

\section{INTRODUÇÃO}

A precipitação é a principal forma de entrada de água no sistema hidrológico o que garante equilíbrio hídrico entre a disponibilidade de água e o consumo pelo ecossistema, interferindo direta e indiretamente nas atividades vitais e econômicas (ALMEIDA et al., 2011).

A região amazônica possui um característico regime hídrico, sendo seis meses de chuva e seis meses de seca (SEDAM, 2010). O período chuvoso apresenta grande variabilidade em relação ao volume de chuva, no entanto é comum que a precipitação nessa região seja de alta intensidade e curta duração, o que caracteriza as chuvas de formação convectiva. Esse tipo de chuva se destaca por gerar volumes significativos de água, influenciando as demais variáveis ambientais, como escoamento superficial e vazão dos rios, podendo provocar cheias nos sistemas de drenagem, grandes prejuízos em áreas agrícolas, como a inundação de terras cultivadas, a erosão do solo, a perda de nutrientes, o assoreamento e a poluição de corpos de água (CECÍlIO et al., 2009; SOUZA et al. 2020).

Diante disso, conhecer a distribuição espacial e temporal da precipitação é de suma importância, principalmente em relação aos eventos extremos de chuvas intensas, pois a ocorrência de tais eventos pode causar situações adversas afetando a sociedade (QAMAR et al., 2017). Sendo assim, para a execução de obras de engenharia em suas variadas áreas, o conhecimento quanto aos eventos extremos se faz indispensável garantindo fator de segurança tanto na execução quanto no funcionamento de tais obras a curto e longo prazo, como destacado por Cruz et al. (2019), Souza et al. (2011) e Souza et al. (2018). 
Uma solução para a caracterização e estimativa das precipitações é a utilização de curvas intensidade-duração-frequência (IDF), que consistem em modelos matemáticos semiempíricos que preveem a intensidade precipitada por meio da duração e da distribuição temporal. Eltz et al. (1992) afirmam que a análise de frequência é uma técnica estatística importante no estudo das chuvas, devido à grande variabilidade temporal e espacial das precipitações pluviais, as quais não podem ser previstas em bases puramente determinísticas.

Esses modelos procuram incorporar as principais características da chuva, permitindo gerar estimativas que visam atender a características específicas da precipitação na localidade para a qual o modelo é concebido, por meio de análises estatísticas, em função de chuvas intensas poderem ser potencialmente ajustadas por meio do emprego de distribuições estatísticas (OLIVEIRA et al. 2008; PEREIRA, DUARTE e SARMENTO, 2017 e ; SOUZA et al. 2020).

A curva IDF de uma dada localidade pode determinar a caracterização de eventos extremos de chuva, possibilitando previsão a longo prazo e auxiliando nas escolhas por melhores alternativas na construção de obras de engenharia (DAMÉ et al., 2006). Diante do exposto, o objetivo deste estudo foi desenvolver a equação IDF do município de Cruzeiro do Sul - AC, com uma série histórica de 14 anos distribuídos entre os anos de 1993 e 2011, com a finalidade de subsidiar estudos hidrológicos.

\section{MATERIAL E MÉTODOS}

A área de estudo é o município de Cruzeiro do Sul, que está localizado no interior do estado do Acre, na porção central da Amazônia, conforme Figura 1, nas coordenadas Latitude $S 7^{\circ} 39^{\prime}$ 54" e Longitude W 72 39' 1" (CIDADE BRASIL, 2018). É o segundo município mais populoso do estado (IBGE, 2018) e está a cerca de 632 quilômetros da capital Rio Branco. 
Os dados obtidos foram registrados pela estação pluviométrica Seringal Belo Monte, sob o código 773001, está localizada nas coordenadas Latitude S $7^{\circ} 23^{\prime}$ 8,16" e Longitude W $73^{\circ} 5^{\prime} 6,00^{\prime \prime}$, na bacia do Rio Amazonas e subbacia 12 do Rio Solimões.

Figura 1 - Mapa de localização da área de estudo

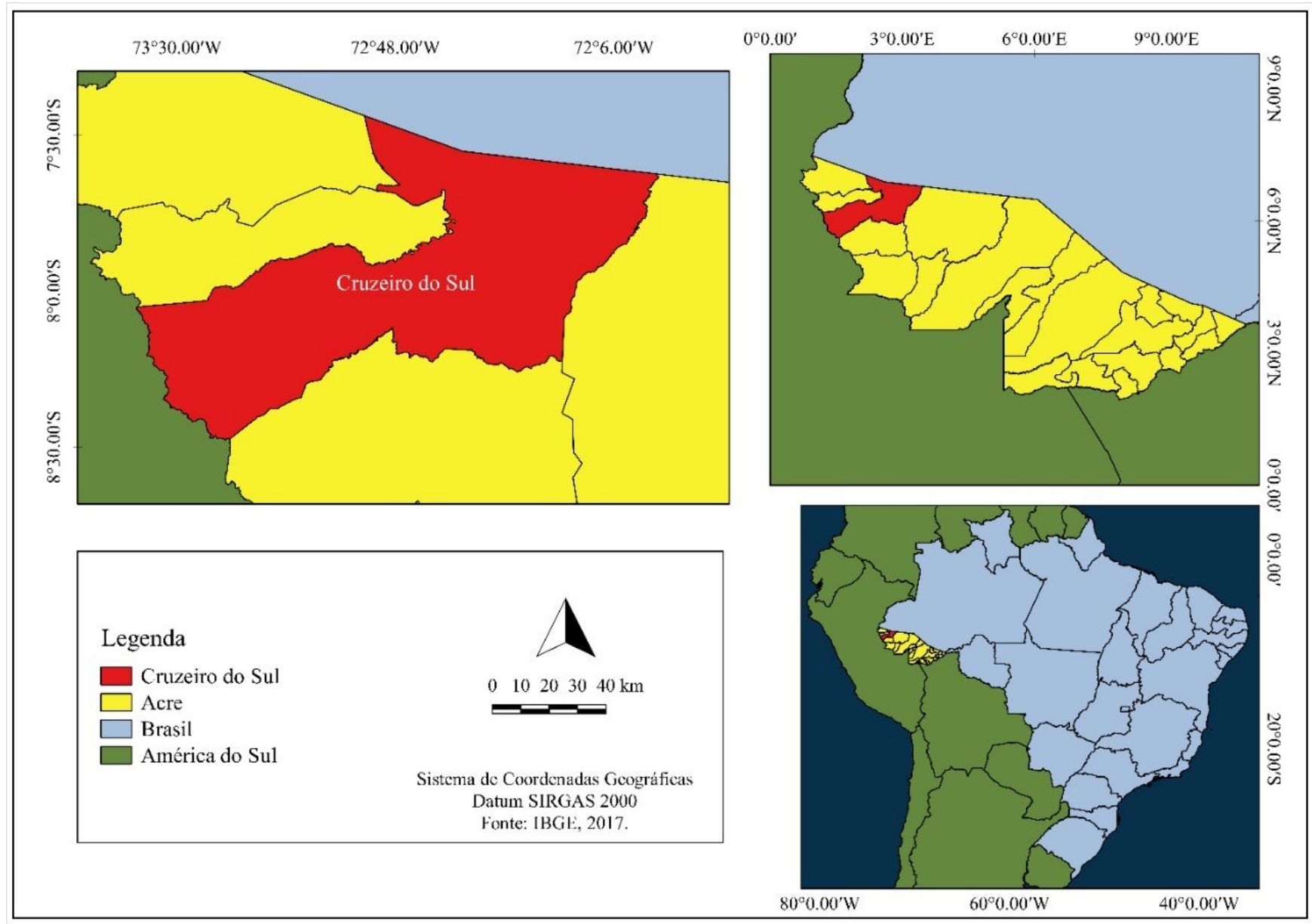

A série histórica de dados de precipitação utilizada no estudo foi obtida no banco de dados disponibilizado pela Agência Nacional das Águas (ANA), sendo a estação pluviométrica que possui o nome Seringal Belo Monte utilizada, a mesma está sob o código 773001 e é localizada nas coordenadas Latitude S $7^{\circ} 23^{\prime} 8,16^{\prime \prime}$ e Longitude W $73^{\circ} 5^{\prime}$ $6,00 "$.

O banco de dados da série compreende do ano 1993 a 2002 e os anos 2005, 2008, 2010 e 2011, totalizando uma série histórica de 14 anos ou 168 meses efetivos para as 
análises realizadas. Levou-se em conta apenas os anos em que os períodos chuvosos não apresentavam falhas, já que o objetivo do estudo necessita de informações desse período.

O critério usado na identificação de um evento extremo de chuva foi o valor máximo diário registrado no decorrer de um ano. No caso, então, foi discriminado um evento para cada ano.

Neste trabalho, optou-se pela utilização da distribuição de Gumbel para modelar e determinar as magnitudes dos tempos de retorno (Tr) - de 1 a 100 anos - dos eventos extremos, máximas diárias, da série histórica dos dados do pluviômetro, visto que tal distribuição estatística é a que melhor se ajusta à região estudada tal como atesta o estudo de Souza, (2019).

O modelo probabilístico de Gumbel (2004), pode ser expresso pela Equação (1):

$$
X=\bar{X}-S\left\{0,45+0,7797 \ln \left[\ln \frac{T r}{T r-1}\right]\right\}
$$

Sendo: $X$ é a precipitação máxima ajustada $(\mathrm{mm}) ; \bar{X}$ é a média dos valores máximos da série histórica de precipitação $(\mathrm{mm})$; S é o desvio padrão dos valores máximos da série histórica de precipitação (mm); e Tr é o período de retorno (anos).

A curva IDF é a relação que a intensidade que um evento de chuva extrema possui com seu tempo de recorrência e a sua duração. Assim para o primeiro termo, o qual é o inverso da probabilidade, pode-se estimar para tempos de retorno pré-definidos intensidades de referência $(X)$ por meio da equação 1. Para o estudo foram adotados os períodos de retorno 2, 10, 25, 50, 75 e 100.

A duração, contudo, é mais complexa de estimação, pois como a maioria dos dados de precipitação obtidos em estações de monitoramento ou dados satélites estão acumulados em duração de 24 horas, é necessário o uso de técnicas de desagregação.

Dentre as técnicas de desagregação algumas que se destacam na literatura são as relações de Bell (1969), que estima a altura da chuva com duração de até 120 min a partir da chuva com duração de uma hora e período de retorno de 10 anos; isozonas, de Torrico (1975); e o método dos coeficientes de desagregação de CETESB (1979), o qual é um método de uso simples e foi concebido ao analisar um quantitativo de 98 postos 
pluviométricos em todo o território brasileiro que possui heterogeneidade climática e mesmo possuindo um caráter regional, este foi comparado com coeficientes de desagregação criados em outros países e descobriu-se diferenças inferiores a 8\% com média de $5 \%$.

Dessa forma a desagregação foi feita usando os coeficientes de CETESB (1979) da Tabela 1 em que para cada período de retorno anteriormente estimado, obteve-se as intensidades com durações de 5, 10, 15, 30, 60, 120, 720 e 1440 minutos.

Tabela 1 - Coeficientes de desagregação da chuva de 24h de duração

\begin{tabular}{lccc}
\hline $\begin{array}{l}\text { Relação entre alturas } \\
\text { Pluviométricas }\end{array}$ & Fator de desagregação & $\begin{array}{c}\text { Relação entre alturas } \\
\text { pluviométricas }\end{array}$ & Fator de desagregação \\
\hline $5 \mathrm{~min} / 30 \mathrm{~min}$ & 0,34 & $1 \mathrm{~h} / 24 \mathrm{~h}$ & 0,42 \\
$10 \mathrm{~min} / 30 \mathrm{~min}$ & 0,54 & $6 \mathrm{~h} / 24 \mathrm{~h}$ & 0,72 \\
$15 \mathrm{~min} / 30 \mathrm{~min}$ & 0,7 & $8 \mathrm{~h} / 24 \mathrm{~h}$ & 0,78 \\
$20 \mathrm{~min} / 30 \mathrm{~min}$ & 0,81 & $10 \mathrm{~h} / 24 \mathrm{~h}$ & 0,82 \\
$25 \mathrm{~min} / 30 \mathrm{~min}$ & 0,91 & $12 / 24 \mathrm{~h}$ & 0,85 \\
$30 \mathrm{~min} / 1 \mathrm{~h}$ & 0,74 & $24 \mathrm{~h} / 1 \mathrm{dia}$ & 1,14 \\
\hline
\end{tabular}

Com as informações das alturas máximas precipitadas para os períodos de retorno e durações desejados, montou-se a equação IDF (intensidade-duração-frequência) para a área de influência da estação pluviométrica em Cruzeiro do Sul, estabelecendo-se as constantes - k, a, b e c - pelo método dos mínimos quadrados (OLIVEIRA et al., 2000; VILLELA e MATTOS, 1975), utilizando na planilha excel a ferramenta Solver, tendo como base a equação IDF geral, Equação (2):

$$
i_{m}=\frac{K \operatorname{Tr}^{a}}{(t+b)^{c}}
$$

Onde, t corresponde à duração da chuva em minutos.

Para a aplicação do Solver na determinação dos parâmetros - K, a, b e c - da equação de chuvas intensas utilizou-se como fator limitante o Erro Médio Normalizado $(E M N)$, estabelecendo uma condição de $E M N=1$, para assegurar um bom ajustamento dos valores (QUEIROZ, 2013; PEREIRA, DUARTE e SARMENTO, 2017). 
No ajuste dos coeficientes e para verificação da eficiência da equação IDF calculou-se as estatísticas do Erro Padrão Médio (EPM) Erro Médio Normalizado (EMN) (Equações 3 e 4), metodologias amplamente utilizadas, em que $\mathrm{N}$ é o número de medidas realizadas, Kp é o coeficiente de valores preditos e $\mathrm{Km}$ é o coeficiente de valores mensurados; porém são consideradas análises tendenciosas por estarem baseadas no erro diferencial (QUEIROZ, 2013).

$$
\begin{aligned}
& E P M=\sqrt{\left(\sum_{i=1}^{N} \frac{\left(K_{p}-K_{m}\right)_{i}^{2}}{N}\right)} \\
& E M N=\frac{100}{N} \sum_{i=1}^{N} \frac{\left(K_{p}-K_{m}\right)}{K_{m}}
\end{aligned}
$$

Portanto, foi utilizado o Erro Médio Multiplicativo, EMM (Equação 5), que de acordo com Moog e Jirka (1998) e Queiroz, Matos e Von Sperling (2015), por estar na base logarítmica, proporciona melhor avaliação do impacto da imprecisão nos dados.

$$
E M M=E X P\left[\frac{\sum_{i=1}^{N} \ln \left(\frac{K_{p}}{K_{m}}\right)}{N}\right]
$$

O coeficiente $\mathrm{R}^{2}$ de regressão é um fator de avaliação da eficiência da equação utilizado no ajuste da função aos pontos observados (SOUZA et al., 2016). O R² consiste na razão entre a soma de quadrados da regressão e a soma de quadrados total, conforme pode ser observado na Equação (6),

$$
R^{2}=\frac{\left(\sum_{i=1}^{n}\left(x_{i}-\bar{x}\right) Y_{i}\right)^{2}}{\sum_{i=1}^{n}\left(x_{i}-\bar{x}\right)^{2} \sum_{i=1}^{n}\left(Y_{i}-\bar{Y}\right)^{2}}
$$

Os dados foram analisados com auxílio dos softwares Microsoft Excel 2010 e Biostat 5.0. Para verificação do ajuste da equação encontrada para cálculo da intensidade foi realizado o teste de Wilcoxon-Mann-Whitney para amostras independentes com a =0,05 após não aderência do teste de normalidade D'Agostino. 


\section{RESULTADOS E DISCUSSÃO}

Ao analisar os dados de precipitação máxima de diária entre os anos de 1993 a 2002 e os anos 2005, 2008, 2010 e 2011 do município de Cruzeiro do Sul no Acre, foi possível observar que os anos 1995 e 2002 corresponderam aos anos com maiores índices de altura precipitada de $111 \mathrm{~mm}$ e $103 \mathrm{~mm}$, respectivamente (Figura 3). Entre esses anos (1995 e 2002) houve as maiores intensidades sendo o tempo de retorno desses fenômenos estimados entre 3 e 8 anos, levando-se em conta a distribuição de Gumbel.

Figura 3 - Série histórica da precipitação máxima de diária por ano, para o município de Cruzeiro do Sul

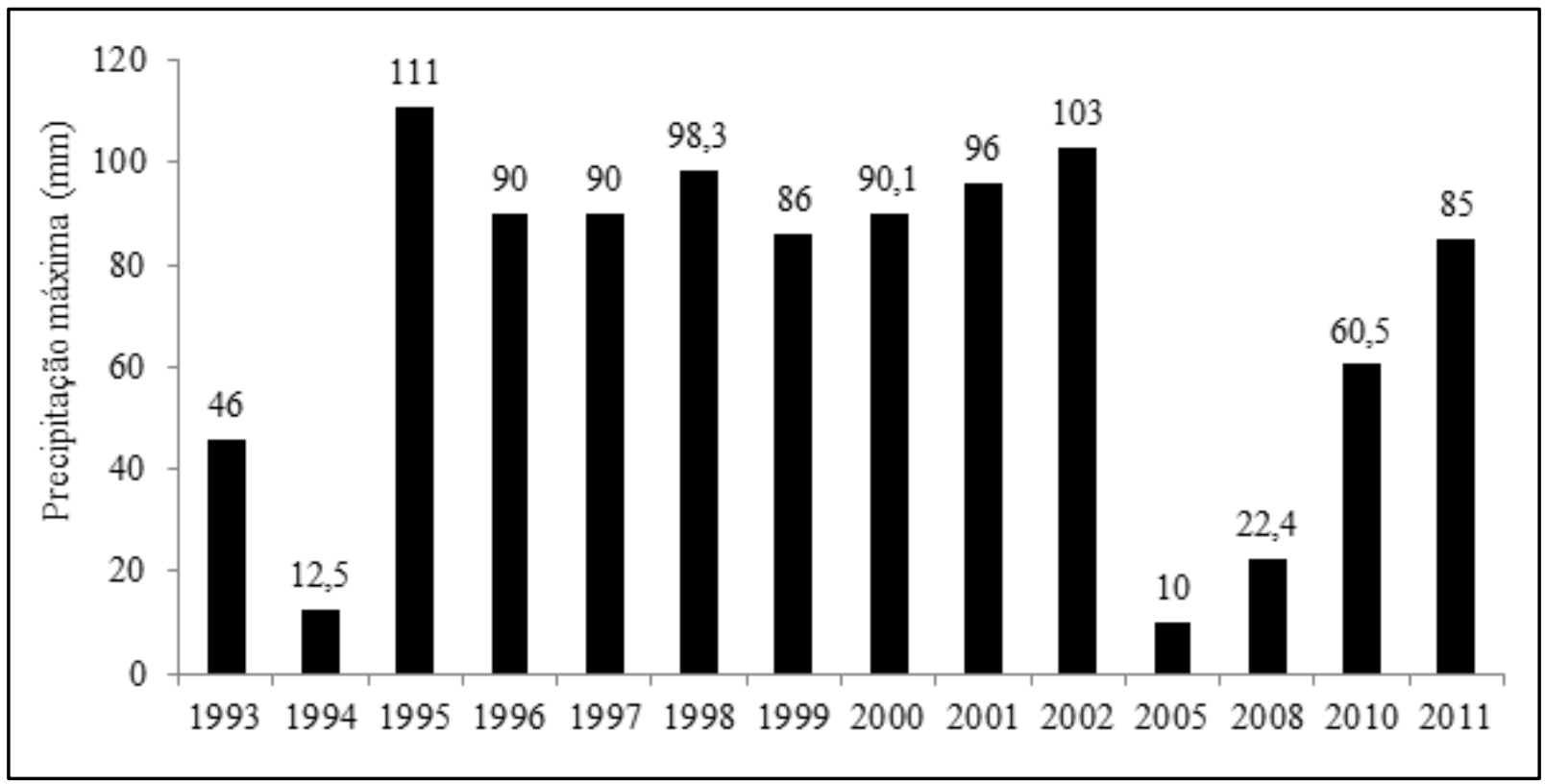

A aleatoriedade nos eventos pode causar situações adversas afetando a sociedade ressaltando-se também que esses altos e variados valores encontrados são característicos da região Amazônica (QAMAR et al., 2017).

A equação desenvolvida no presente estudo (Equação 7) para estimativa da intensidade das precipitações máximas, utilizou como base as durações de 5, 10, 15, 20, $30,60,120,480,600,720$ e 1.440 minutos e os tempos de retorno de $2,5,10,20,50$ e 100 
anos. Sendo assim, a Equação 10 abrange ampla faixa de aplicação por causa da amplitude característica dos intervalos.

$$
i_{m}=\frac{615,9604 \cdot T r^{0,3268}}{(t+9,9131)^{0,7685}}
$$

De maneira geral a Equação 9 apresenta o típico comportamento das curvas IDF, que pode ser melhor visualizado pela Figura 4, na qual é visível a relação de proporcionalidade inversa entre a intensidade da chuva e sua duração, conforme constatado por diversos estudos (PEREIRA et al., 2007 apud SOUZA et al., 2013; SOUZA et al., 2015; PEREIRA, DUARTE e SARMENTO, 2017), esta relação exprime que quanto menor a duração da chuva maior será sua intensidade.

Figura 4 - Curvas IDF do município de Cruzeiro do Sul, Acre

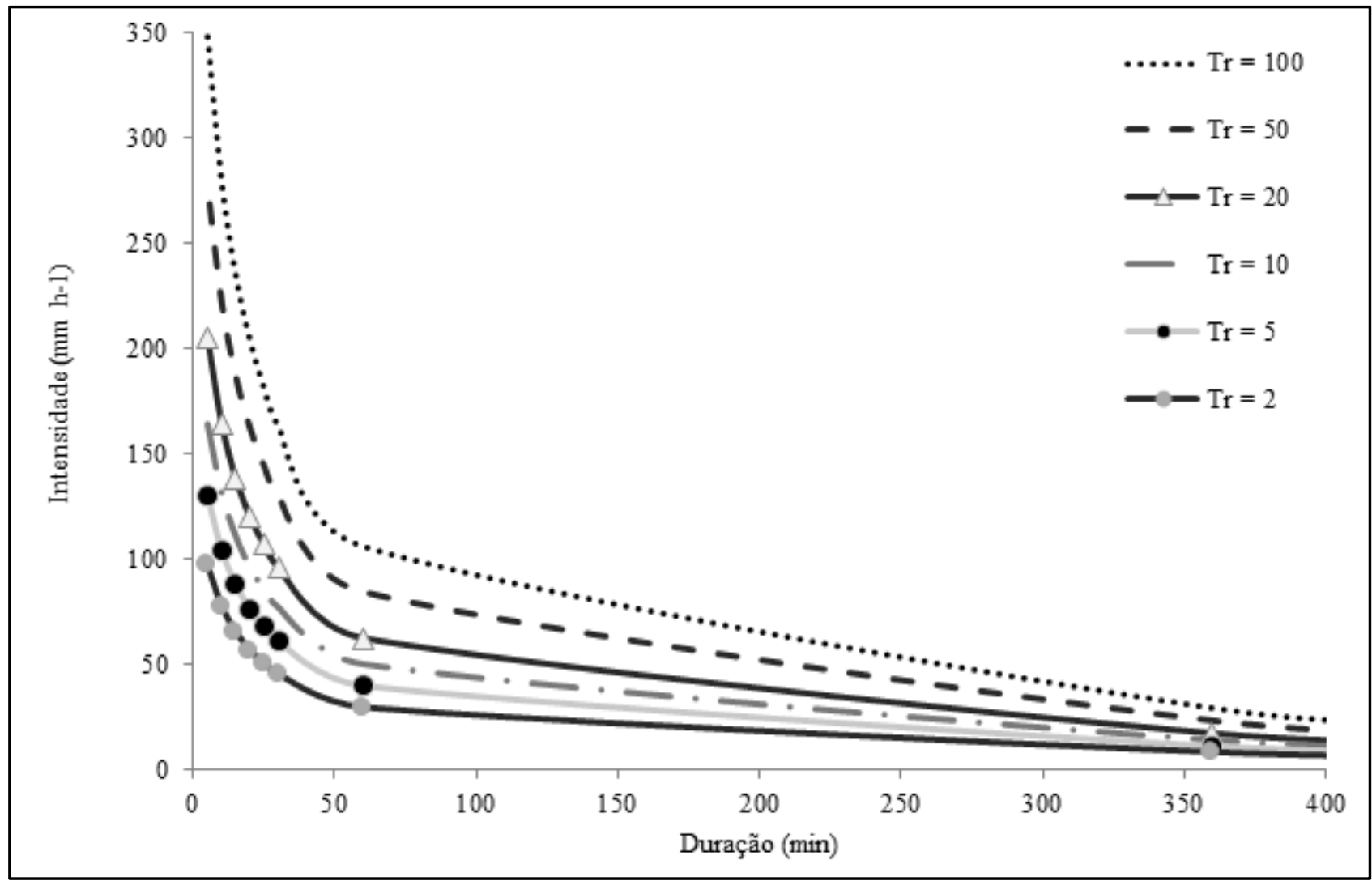


Ao analisar o EPM (Equação 3) e o EMN (Equação 4) foram encontrados os valores de 28,24 e $28,07 \%$, respectivamente, em que para que haja um bom ajustamento os valores devem estar próximos a 0 , no entanto são consideradas análises tendenciosas por estarem baseadas no erro diferencial.

O EMM (Equação 5), é a metodologia que proporciona a melhor avaliação do impacto da imprecisão nos dados e, apesar das outras serem amplamente utilizadas, essa foi a metodologia adotada para as análises, resultando em um EMM igual a 1, corroborando assim um bom ajustamento aos valores.

O coeficiente de determinação $\left(r^{2}\right)$ da equação dos dados observados resultou em 0,9551, ou seja $95,51 \%$ dos dados de log (Tr) podem ser explicados pela precipitação máxima anual. Ao analisar os dados de intensidade obtidos a partir da equação encontrada para região em relação aos dados observados pela estação pluviométrica foi possível obter uma correlação com intensidade forte onde $r=0,84$ e não foi constatada diferença estatistica significativa entre os valores estimados e observados com $p$ valor $=0,89$ o que mostra a eficiência da equação.

\section{CONCLUSÕES}

Ao analisar os dados de intensidade obtidos a partir da equação encontrada para o Cruzeiro do Sul em relação aos dados observados pela estação pluviométrica foi possível obter uma correlação forte e não houve constatação de diferença estatística significativa entre os valores estimados e observados, mostrando a eficiência da equação.

Foi possível observar que os anos 1995 e 2002 corresponderam aos anos com maiores índices de altura precipitada de $111 \mathrm{~mm}$ e $103 \mathrm{~mm}$, respectivamente. A equação encontrada para a área de estudo apresenta o típico comportamento das curvas IDF com relação de proporcionalidade inversa entre a intensidade da chuva e sua duração. 
A curva IDF de uma dada localidade, pode determinar a caracterização de eventos extremos de chuva, possibilitando previsão a longo prazo e auxiliando nas escolhas por melhores alternativas na construção de obras de engenharia.

\section{REFERÊNCIAS}

ALMEIDA, A. Q.; RIBEIRO, A.; PAIVA, Y.G.; RASCON, N. J. L.; LIMA, E. P. Geoestatística no estudo de modelagem temporal da precipitação. 2011. Revista Brasileira de Engenharia Agrícola e Ambiental, v. 15, n. 4, p. 354-358.

ANA. Hidroweb. 2018. Disponível em:

http://www.snirh.gov.br/hidroweb/publico/mapa_hidroweb.jsf . Acesso em 29 de nov.

CECÍlIO, R. A.; XAVIER, A.C.; PRUSKI, F. F.; HOLLANDA, M. P.; PEZZOPANE, J. E. M. Avaliação de interpoladores para os parâmetros das equações de chuvas intensas no Espírito Santo. AmbiAgua, Taubaté, v. 4, n. 3, p. 82-92, 2009.

CIDADE BRASIL. Município de Cruzeiro do Sul. 2018. Disponível em:

https://www.cidade-brasil.com.br/municipio-cruzeiro-do-sul.html . Acesso em 29 de nov.

COMPANHIA DE TECNOLOGIA DE SANEAMENTO AMBIENTAL - CETESB. Drenagem urbana: manual de projeto. São Paulo: CETESB, 1979. 476p.

CRUZ, J. S. et.al. Equações de chuvas intensas com dados CPC morphing technique (CMORPH) para o município de Altamira - PA. Irriga, Botucatu, v. 24, n. 1, p. 192-207, 2019.

ELTZ, F. L. F.; REICHERT, J. M.; CASSOL, E. A. Período de retorno de chuvas em Santa Maria, RS. R. Bras. Ci. Solo, v. 16, n. January 1992, p. 265-269, 1992.

DAMÉ, R. C. F. et al. Comparação entre curvas intensidade-duração-frequência de ocorrência de precipitação obtidas a partir de dados pluviográficos com àquelas estimadas por técnicas de desagregação de chuva diária. Revista Brasileira de Agrociência, Pelotas, v. 12, n. 4, p.505-509, 2006.

IBGE. Município Cruzeiro do Sul. 2018. Disponível em:

https://cidades.ibge.gov.br/brasil/ac/cruzeiro-do-sul/panorama . Acesso em 29 de nov.

MOOG, D. B.; JIRKA, G. H. Analysis of Reaeration Equations Using Mean Multiplicative Error. 1998. J. Envion. Eng. 124 (2): p. 104-110.

QAMAR, M. U. et al. Rainfall Extremes: a Novel Modeling Approach for Regionalization. Water Resources Management, v.31, n.6, p.1975-1994, 2017.

QUEIROZ, F. M. de. Estudo da reaeração da água em canal com fundo deslizante. 2013. Tese (Doutorado em Scentiae) - Universidade Federal de Viçosa, Viçosa. 
QUEIROZ, F. M. de; MATOS, A. T. de; VON SPERLING, M. Estimativa do coeficiente de rearação da água em canal raso de fundo deslizante. 2015. Eng. Sanit. Ambient. v. 20. n. 1. jan/mar. P. 79-88.

OLIVEIRA, L. F. C. de et al. Estimativas das equações de chuvas intensas para algumas localidades o Estado de Goiás pelo método da desagregação de chuvas. 2000. Pesquisa Agropecuária Tropical. 30 (1): 23-27, jan/jun.

OLIVEIRA, L. F. C. et al. Métodos de estimativa de precipitação máxima para o Estado de Goiás. Revista Brasileira de Engenharia Agrícola e Ambiental, v.12, n.6, p. 620-625, 2008.

PEREIRA, D. C.; DUARTE, L. R.; SARMENTO, A. P. Determinação da curva de intensidade, duração e frequência do município de Ipameri - Goiás. 2017. Revista Eletrônica de Engenharia Civil. v. 13. n.2. p. 233-246. jul/dez.

SEDAM. 2010. Boletim Climatológico de Rondônia - 2010. Porto Velho: Secretaria de Estado do Desenvolvimento Ambiental. Disponível em: http://www.sedam.ro.gov.br. Acesso em 10 dez. 2018.

SOUZA, V. A. S. de et al. Analysis of extreme rainfall events in the city of Ji-Paraná, Rondônia. 2011. Revista Pesquisa \& Criação. v. 10. n.2. jun/dez. p. 139-151.

SOUZA, V. A. S. de et al. Análise das chuvas intensas no município de Guajará-Mirim, Rondônia. In: XIX Simpósio Brasileiro de Recursos Hídricos, Maceió, 2018.

SOUZA, V. A. S. de et al. Equação de intensidade-duração e frequência de chuvas para o município de Ouro Preto do Oeste, Rondônia. In: SIMPÓSIO BRASILEIRO DE RECURSOS HÍDRICOS (SBRH), 10., 2013b, Bento Gonçalves/RS. Anais. Associação Brasileira de Recursos Hídricos: ABRH, 2013. p. 1-6. 17-22 de nov.

SOUZA, V. A. S. de et al. Equação de Intensidade-Duração-Frequência (IDF) de chuvas de Rolim de Moura - RO. 2015. Revista Brasileira de Ciências da Amazônia. v. 4, n. 1, p. 1-12.

SOUZA, V. A. S. de et al. Determining IDF equations for the state of Rondônia. 2016. Revista Brasileira de Climatologia. v. 18, jan/jun. ISSN: 1980-055x (Impressa) 2237-8642 (Eletrônia).

SOUZA, V. A. S. de et al. Extreme rainfall events in Amazonia: The Madeira river basin. 2020. Remote Sensing Applications: Society and Environment. v. 18.

SOUZA, V. A. S. Padrões e tendências hidroclimáticas de chuva identificados por sensoriamento remoto na bacia do Rio Madeira - Amazônia. 2019. Tese de doutorado (Doutorado em Engenharia Civil). Universidade Federal do Rio de Janeiro, Rio de Janeiro, 2019.

TORRICO, J. J. T. Práticas hidrológicas. Rio de Janeiro: Transcom, 1975.

VILLELA, S. M. e MATTOS, A. Hidrologia Aplicada. 1975. McGraw-Hill do Brasil, São Paulo, SP. 245p. 


\section{CONTRIBUIÇÕES DE AUTORIA}

\section{1 - Nayara dos Santos Albrigo}

Fundação Universidade Federal de Rondônia, Estudante Engenharia Ambiental e Sanitária https://orcid.org/0000-0003-1196-4641 - nayara.santosalbrigo95@gmail.com

Contribuição: Metodologia

\section{2 - Maylla Tawanda dos Santos Pereira}

Fundação Universidade Federal de Rondônia, Estudante Engenharia Ambiental e Sanitária https://orcid.org/0000-0002-9058-0947 - maylla.lifeplay@gmail.com

Contribuição: Concepção

\section{3 - Nelma Tavares Dias Soares}

Fundação Universidade Federal de Rondônia, Estudante Engenharia Ambiental e Sanitária https://orcid.org/0000-0001-5155-3607 - ntds08@gmail.com

Contribuição: Recursos

\section{4 - Gleibson de Souza Andrade}

Fundação Universidade Federal de Rondônia, Estudante Engenharia Ambiental e Sanitária https://orcid.org/0000-0002-1803-5469 - gleybson-desouza@hotmail.com

Contribuição: Escrita - revisão e edição

\section{5 - Vinicius Alexandre Sikora de Souza}

Universidade Federal do Rio de Janeiro, Doutor

http://orcid.org/0000-0002-5902-6139 - vass1000@gmail.com

Contribuição: Escrita - revisão e edição

\section{6 - Ana Lúcia Denardin da Rosa}

Fundação Universidade Federal de Rondônia, Doutora

https://orcid.org/0000-0003-0470-5115 - analucia@unir.br

Contribuição: Supervisão

\section{COMO CITAR ESTE ARTIGO}

ALBRIGO, N. S.; PEREIRA, M. T. S.; SOARES, N. T. D.; ANDRADE, G. S.; SOUZA, V. A. S.; ROSA, A. L. D. Determinação da curva de intensidade-duração-frequência do município de Cruzeiro do Sul - Acre. Ciência e Natura, Santa Maria, v. 43, e30, p. 1-13, 2021. Disponível em: https://doi.org/10.5902/2179460X38301. Acesso em: dia, mês (abreviado), ano. 